THE EQUILIBRIUU BETWEEN BIVALENT AND QUADRIVALENT PAILADIUL

AND CHLORINE IN EYDROCHLOKIC ACID SOLUTION

\author{
Thesis by \\ Homer Bigelow Wellman
}

In partial fulfillment of the requirements

for the degree of Doctor of Philosophy

California Institute of Technology

Pasadena, California

1930 
[Reprint from the Journal of the American Chemical Society, 52, 985 (1930).]

[Contribution from Gates Chemical, Laboratory, California Institute of TECHNOL,OGY, No. 244]

\title{
THE EQUILIBRIUM BETWEEN BIVALENT AND QUADRIVALENT PALLADIUM AND CHLORINE IN HYDROCHLORIC ACID SOLUTION
}

BY HOMER B. WeLLMAN

\author{
RECEIVED DECEMBER 9, 1929 \\ PUblished March 6, 1930
}

\section{Introduction}

The equilibrium between palladous and palladic chlorides and chlorine in hydrochloric acid solution seems not to have been previously measured, and no physico-chemical studies have been made as to the state in which bivalent and quadrivalent palladium exist in chloride solutions. It is well known, however, that from such solutions salts of the types $\mathrm{R}_{2} \mathrm{Pd}$ $\mathrm{Cl}_{4}$ and $\mathrm{R}_{2} \mathrm{PdCl}_{6}$ separate, and it is probable that in such solutions the palladium exists mainly or wholly in the form of the corresponding ions $\mathrm{PdCl}_{4}{ }^{--}$and $\mathrm{PdCl}_{6}{ }^{--}$. In that case the equilibrium to be here considered would be expressed by the simple equation: $\mathrm{PdCl}_{4}^{--}+\mathrm{Cl}_{2}=$ $\mathrm{PdCl}_{6}--$.

It was necessary, however, to consider the possible existence of trivalent palladium in the solution, for complex salts of this valence have been prepared. ${ }^{1}$ Therefore, it was thought advisable in some of the experiments to fix the concentration of quadrivalent palladium by saturating the solutions with the slightly soluble potassium chloropalladate $\left(\mathrm{K}_{2} \mathrm{PdCl}_{6}\right)$, and to estimate the concentration of trivalent palladium in these solutions by determining the total oxidizing chlorine. Accordingly, one of the steps in this investigation consisted in determining the solubility of potassium chloropalladate. Solutions of palladous chloride in normal hydrochloric acid containing known amounts of potassium chloride were saturated with.chlorine at atmospheric pressure, whereby all of the palladium is oxidized to the quadrivalent state. The solubilities of potassium

${ }_{1}^{1}$ Wöhler and Martin, Z. anorg. Chem., 57, 398-413 (1907), by treating the hydrochloric acid solution of palladous chloride with cesium and rubidium chlorides and chlorine, prepared the compounds $\mathrm{Cs}_{2} \mathrm{PdCl}_{5}$ and $\mathrm{Rb}_{2} \mathrm{PdCl}_{6}$, which they found to be more unstable than the corresponding quadrivalent compounds. They failed to prepare $\mathrm{K}_{2} \mathrm{PdCl}_{5}$ by this method, but with considerable difficulty they obtained some of this compound by treating the oxide, $\mathrm{Pd}_{2} \mathrm{O}_{3}$ (formed at the anode in electrolyzing palladous nitrate), with potassium chloride and dry hydrogen chloride in ether. 
chloropalladate in these solutions were then found by gravimetric determination of the total palladium.

A series of measurements of the equilibrium of the oxidation reaction was made, first in the presence of potassium chloropalladate. The solutions were saturated both with chlorine at some definite small partial pressure and with the solid chloropalladate. Chlorine diluted with nitrogen was passed through the palladium solution and then through a second solution identical with the first, except that it contained no palladium. The concentration of the chlorine dissolved in this second solution was found by iodimetric titration. The sum of the concentrations of the free chlorine and of the combined oxidizing chlorine (corresponding to the quadrivalent and to any trivalent palladium) in the solution was measured by titrating it with hydrazine sulfate and determining the endpoint electrometrically. A comparison of this observed concentration of oxidizing chlorine with the concentration of quadrivalent palladium as calculated from the results of the previous determinations of the solubility of potassium chloropalladate showed that there is little, if any, trivalent palladium in the solutions here investigated.

Finally, more precise measurements of the equilibrium of the oxidation reaction were made with normal hydrochloric acid solutions containing known quantities of palladium chloride, but no potassium chloride, the concentrations of free and of combined chlorine being determined in the same way as before. In order to determine the effect of hydrogen ion and of chloride ion on the equilibrium, some measurements were made also in $0.3 \mathrm{~N}$ hydrochloric acid solutions and in solutions $0.7 \mathrm{~N}$ in sodium chloride and $0.3 \mathrm{~N}$ in hydrochloric acid.

This research was suggested by Professor A. A. Noyes, and to him and to Dr. Don M. Yost the author is indebted for many helpful suggestions made during the investigation.

\section{Preparation of the Substances and Solutions}

The stock solutions of palladous chloride used in these experiments were prepared from pure palladium foil. This was dissolved in aqua regia, and the nitric acid was then destroyed by repeated evaporations with hydrochloric acid. The residue of palladous chloride was heated to $300^{\circ}$ until all of the free acid was driven off, and the solution was made up by dissolving this salt in a standard $1 \mathrm{~N}$ hydrochloric acid solution and filtering to remove any metal formed by decomposition. The palladium content of the stock solution was determined by gravimetric analyses.

The palladium metal was carefully purified before preparing the palladium chloride solution which was used in the final experiments on the equilibrium of the oxidation reaction. About $2.5 \mathrm{~g}$. of the foil was converted to palladous chloride as before, and the slightly acid solution of this salt was treated with an excess of mercuric cyanide, whereby the palladium was precipitated as the flocculent, cream-colored cyanide, $\operatorname{Pd}(C N)_{2}$. This was allowed to settle for a few hours, and was then washed several times by decantation; it was not freed from mercury owing to the difficulty of filtering the precipitate. 
Ignition of the precipitate first in oxygen then in hydrogen at $800^{\circ}$ volatilized the mercury, leaving the palladium as metal. This was again converted to the chloride; and by treating the solution with ammonium hydroxide and hydrochloric acid the palladium was twice precipitated as palladosammine chloride, $\mathrm{Pd}\left(\mathrm{NH}_{3}\right)_{2} \mathrm{Cl}_{2}$, which was washed with dilute hydrochloric acid. The chloride solution obtained by boiling this precipitate in acid was saturated with potassium chloride and chlorine; and the potassium chloropalladate was filtered off and ignited in hydrogen. From the pure palladium metal so obtained the stock solution was prepared and standardized as previously described.

All of the dilute thiosulfate solutions required for the iodine titrations were made up as needed from a stock solution of $0.1 \mathrm{~N}$ sodium thiosulfate. This was prepared from freshly boiled distilled water and a high grade of thiosulfate, and to each liter of the solution $0.2 \mathrm{~g}$. of sodium carbonate was added as recommended by Kolthoff ${ }^{2}$ to prevent decomposition. This solution was standardized against standard solutions of potassium permanganate and of potassium dichromate according to the methods described by Bray and Miller. ${ }^{3}$ In four months the stock solution so prepared had changed in concentration less than $0.1 \%$. It was also found that the ten-fold or hundred-fold diluted solutions, if used within an hour of the time of their preparation, had the corresponding strength within $0.1 \%$.

The solution of hypochlorous acid used in the electrometric titrations was prepared by passing chlorine gas into a suspension of mercuric oxide in water, and by twice distilling the solution over mercuric oxide to free it from chloride ion. By diluting the distillate, a solution $0.01 \mathrm{~N}$ in $\mathrm{HClO}$ was thus obtained.

The hydrazine sulfate solution was prepared from the recrystallized salt, and was diluted to approximately the same strength as the hypochlorous acid.

\section{Methods of Analysis}

Determination of Total Palladium.-The total palladium present in the solutions was determined by precipitating it as the palladous salt of dimethylglyoxime. ${ }^{4}$ The palladium precipitate is too soluble to be washed with hot water, since $1 \mathrm{mg}$. dissolves in 200-300 cc. of boiling water; but when $0.25 \mathrm{~g}$. of palladium was precipitated from a liter of cold $0.5 \mathrm{~N}$ hydrochloric acid, only $0.3 \mathrm{mg}$. was found in the filtrate. It was found that the compound is not hygroscopic, can be dried to constant weight at $110^{\circ}$, and does not decompose at $130^{\circ}$. In this work the palladium was, therefore, determined by weighing the dry dimethylglyoxime precipitate. The palladium determined by evaporating portions of the stock solution in weighed porcelain boats and igniting the residue in hydrogen checked within $0.5 \%$ with that calculated from corresponding analyses by this dimethylglyoxime method, although only $50 \mathrm{mg}$. of palladium was used for each analysis. The dimethylglyoxime method has the additional advantage that the compound contains only a fairly small proportion $(31.68 \%)$ of palladium. The flocculent light yellow precipitate, which has a composition like that of the familiar nickel compound; is not difficult to filter through perforated crucibles (unless there is more than $0.25 \mathrm{~g}$. of it).

The procedure adopted for the palladium analysis was as follows. Add a solution of about $0.2 \mathrm{~g}$. of dimethylglyoxime in $100 \mathrm{cc}$. of boiling water to the palladium solution

2 Kolthoff, C. A., 13, $3103^{2}$ (1919).

3 W. C. Bray and Miller, Trrs Journal, 46, 2204 (1924).

${ }^{4}$ Wunder and Thüringer, Z. anal. Chem., 52, 101-104, 660-664 (1913); Davis, C. A., 16, $2280^{4}$ (1922); Cooper, C. A., 20, $1042^{6}$ (1925). These investigators all converted the precipitate by ignition to metallic palladium. 
diluted to 200-300 cc., boil the mixture and allow it to stand overnight; then filter it through a weighed crucible and wash the precipitate with cold water until free from chloride ion; dry it at $110^{\circ}$ for an hour and weigh. Subsequent weighings agreed nearly always within $0.2 \mathrm{mg}$. and separate analyses usually agreed also within $0.2 \mathrm{mg}$.

Determination of the Free Chlorine.-The chlorine dissolved in the acid solution through which the diluted gas was passed in the equilibrium experiments was determined by adding potassium iodide and titrating the liberated iodine with $0.001-0.005 \mathrm{~N}$ thiosulfate using starch as indicator. A known volume of the solution was run from the apparatus into $600 \mathrm{cc}$. of distilled water containing $2 \mathrm{~g}$. of potassium iodide. A blank titration was always made and the correction applied, but the indistinctness of the endpoint caused considerable difficulty, especially when $0.001 \mathrm{~N}$ thiosulfate was used for the titration.

Determination of the Total Oxidizing Chlorine.-To determine the combined oxidizing chlorine and the free chlorine dissolved in the palladium solutions, only the electrometric titration could be employed. A known volume of chlorinated palladium chloride solution was run out of the apparatus into a mixture of $200 \mathrm{cc}$. of water containing $2 \mathrm{~g}$. of potassium bromide with such a volume of the standard $0.01 \mathrm{~N}$ hydrazine sulfate solution that there was little or no excess of bromine liberated (not more than 0.03 milliequivalent). A platinum plate was placed in the solution; this was connected with a calomel electrode (with $0.5 \mathrm{~N}$ potassium chloride), and the end-point was determined with the aid of an ordinary potentiometer when more hydrazine sulfate was added or the solution was titrated back with hypochlorous acid. The end-point was determined several times with the same solution in order to standardize the hypochlorous acid at the same time. Until the end-point was reached, the potential remained a little above 0.7 volt; but a single drop of $0.01 \mathrm{~N}$ hydrazine solution would then reduce this potential by 0.06 volt, while any further addition caused a far more gradual change in potential. In some cases the same palladium solution was after this titration used for determining the total palladium by a dimethylglyoxime precipitation.

Standardization of the Hypochlorous Acid.-The hypochlorous acid solution was standardized by adding potassium iodide and titrating with $0.01 \mathrm{~N}$ thiosulfate solution. It was then at once used in the subsequent electrometric titrations.

Standardization of the Hydrazine Solution.-Bray and Cuy ${ }^{5}$ have shown that the reaction of hydrazine sulfate with bromine is quantitative in acid or neutral solution but that with hypochlorous acid one must add phosphate to buffer the solution and thus prevent loss of chlorine. It was found, however, that by adding potassium bromide to the hydrazine sulfate solution before running in the hypochlorous acid the loss of chlorine or bromine was avoided, for the results by this method of standardization checked with those obtained by the method of Bray and Cuy with bromine solution. Moreover, it was found that palladium salts do not interfere: the quadrivalent palladium is readily reduced to the bivalent state by the hydrazine sulfate, but there is no further reduction at room temperature in the presence of the bromide and some acid.

The procedure adopted for the standardization of the hydrazine was therefore as follows: $25 \mathrm{cc}$. of the solution was pipetted into $100 \mathrm{cc}$. of a $0.01 \mathrm{~N}$ hydrochloric acid solution containing $1 \mathrm{~g}$. of potassium bromide. Then into this solution was pipetted $25 \mathrm{cc}$. of freshly standardized hypochlorous acid solution which was a little stronger than the hydrazine solution. The vessel was stoppered, and after five minutes $1 \mathrm{~g}$. of potassium iodide was added, and the iodine liberated by the excess of bromine was titrated with $0.01 \mathrm{~N}$ thiosulfate solution. To check the results obtained by this method, the excess of bromine in some cases was titrated electrometrically with hydrazine sulfate, whereby the same results were obtained.

5 W. C. Bray and Cuy, Thrs Journal, 46, 858-875 (1924). 


\section{Apparatus and Procedure}

The Solubility Experiments. - A water thermostat regulated to $25.00 \pm 0.02^{\circ}$ was used for all the experiments. The solubility apparatus consisted; as shown in Fig. 1, of a glass-stoppered 100-cc. round-bottomed flask having a tube fused into the bottom and a side-tube fused into the neck. The chlorine gas was passed from an iron cylinder first through $1 \mathrm{~N}$ hydrochloric acid in the thermostat, then through four such flasks in series, so that four separate experiments were carried on simultaneously. The chlorine entered the flask at I and escaped from it at O. As connectors were used rubber tubes which had been previously

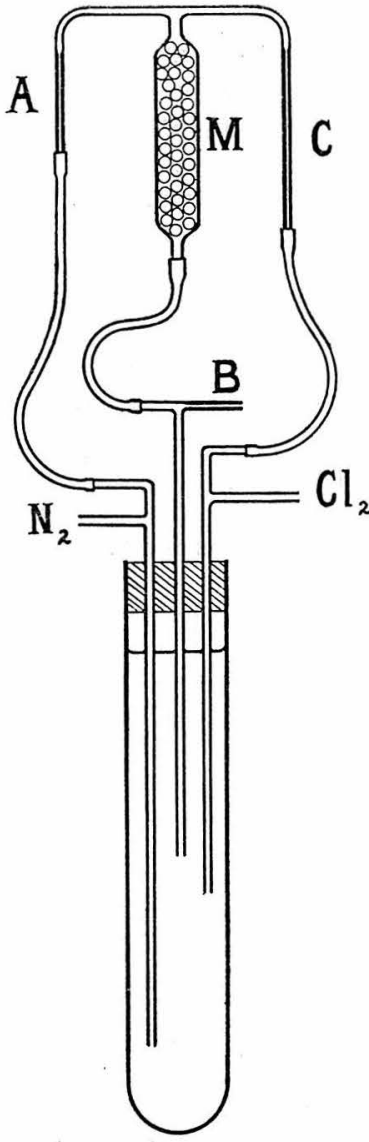

Fig. 2. soaked in hot paraffin to prevent their being acted upon by the gas. Samples were removed from time to time for the palladium analysis.

The solutions were made up by pipetting into each flask various volumes of the stock solutions of palladous chloride, potassium chloride and hydrochloric acid, and by adding also about $0.25 \mathrm{~g}$. of finely powdered potassium chloropalladate.

To withdraw a sample the stopper was removed and the liquid was drawn quickly from the flask into a partially evacuated vessel

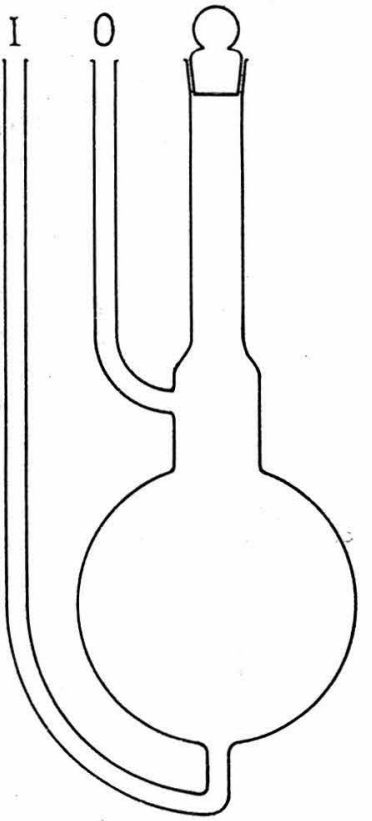

Fig. 1. through a glass tube containing a plug of asbestos fibers held in place by glass wool and by constrictions in the tube. In this way the solution remained saturated with chlorine until it had passed through the filter. Of this filtered solution $25 \mathrm{cc}$. was pipetted into a beaker, the chlorine boiled off, and the palladium precipitated with dimethylglyoxime and weighed. Fresh samples were often withdrawn from the flask several days later, in order to show whether the solution had come to equilibrium with the solid phase.

Preparation of the Chlorine-Nitrogen Mixture.-In all the measurements of the equilibrium of the oxidation reaction it was necessary to dilute with nitrogen the chlorine bubbled through the equilibrium mixtures, since otherwise the palladium would have been completely oxidized. The apparatus shown in Fig. 2 served to produce the gas mixture and to keep it at constant composition within $1-2 \%$ during an entire experiment, which lasted in some cases as long as five days. Chlorine from the cylinder entered one arm of a T-tube, the stem of which dipped about half a meter below the surface of concentrated sulfuric acid in a tall glass cylinder $5 \mathrm{~cm}$. $\mathrm{n}$ diameter. The other arm of the T-tube was connected through a very fine capillary 
tube $\mathrm{C}$ (which served as a resistance to the flow of chlorine) with the mixing chamber $M$, consisting of a 50 -cc. glass tube filled with beads. In a similar way nitrogen was led from

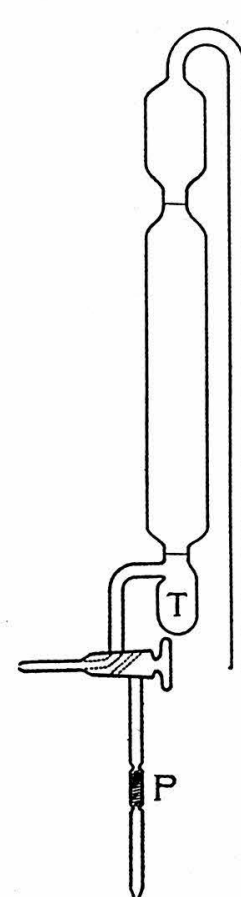

Fig. 3. the tank into the mixing chamber. The mixed gases leaving the chamber then entered one arm of a third T-tube whose stem dipped below the surface of the acid only far enough to give the pressure needed to drive the gases through the third capillary $B$ into the equilibrium vessel at the desired rate. The partial pressure of chlorine was thus controlled by duly selecting the three capillaries, $A, B, C$, and by raising or lowering the tubes dipping into the sulfuric acid.

Measurement of the Reaction Equilibrium.-The first equilibrium measurements were made in the presence of solid potassium chloropalladate. The equilibrium mixtures were made up in just the same way as for the simple solubility determinations. The apparatus consisted of two calibrated 50-cc. pipets. The diluted chlorine was passed first through the palladous chloride solution in the one pipet, and next through a solution in the second pipet which contained acid and potassium chloride at the same concentrations, but no palladium. The first of these pipets, shown in Fig. 3, was provided with a trap $T$ to catch most of the solid salt, and with an exit tube containing a plug, $\mathrm{P}$, of asbestos fibers to retain the finer particles. The gas was allowed to flow for several days through the apparatus kept in the thermostat. The pipets were then removed from the thermostat one at a time and were inverted while leaving the gas line attached, so that the measured volume of solution could be drawn off without changing the chlorine concentration. These samples were titrated as described above. Since some of the palladium crystallized out as potassium chloropalladate, a gravimetric analysis for palladium had to be made. For this purpose the solution was used in which the chlorine content had already been determined by the electrometric titration with hydrazine.

For the final experiments in which no potassium chloride was added and no solid potassium chloropalladate separated, an all-glass apparatus was designed as shown in Fig. 4. The vessel W contained hydrochloric acid of the strength prevailing in the reaction mixture. The small 50-cc. bulb $\mathrm{P}$ in the middle contained the solution of palladous chloride in hydrochloric acid, and the two 100-cc. bulbs AA at the sides contained a solution of the pure acid of the same concentration. The diluted chlorine was passed through the apparatus in the thermostat for three hours only, for experiments in which the equilibrium was approached from the other side by passing at first pure chlorine and then the diluted gas, showed that equilibrium is reached within two hours with

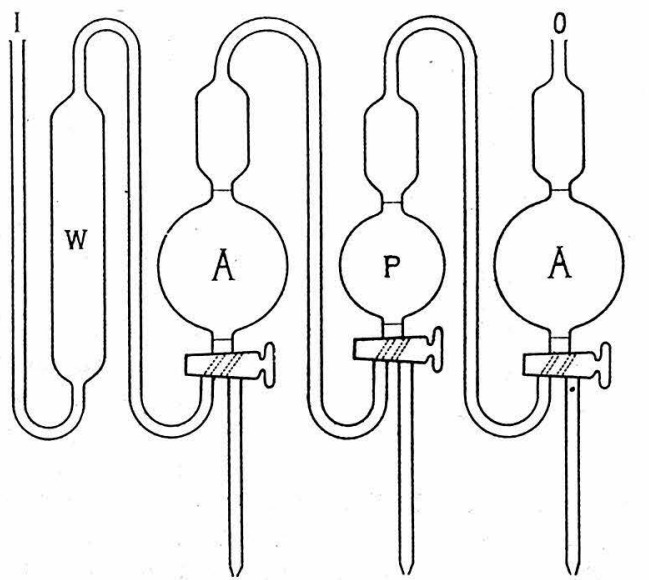

Fig. 4.

this apparatus. To remove the samples for titration, the chlorine tube was attached to the other end $O$ of the apparatus, so that the same partial pressure of chlorine was main- 
tained above each surface while the solutions were drawn off. The two pure acid solutions were titrated for the dissolved chlorine, and the average value was taken.

\section{The Solubility of Potassium Chloropalladate}

The results of the solubility measurements are given in Table I. The solution always was $1.0 \mathrm{~N}$ in hydrochloric acid and saturated with chlorine at the atmospheric pressure (about $740 \mathrm{~mm}$.) at $25^{\circ}$.

TABLE I

Solubility of Potassium Chloropalladate at $25^{\circ}$ in Normal Hydrochloric Acid Saturated with Chlorine and Containing Potassium Chloride

\begin{tabular}{|c|c|c|c|c|c|c|c|c|c|}
\hline \multirow{2}{*}{$\begin{array}{c}\text { Expt. } \\
\text { no. } \\
1\end{array}$} & $\begin{array}{l}\text { Time, } \\
\text { days }\end{array}$ & $\begin{array}{l}\text { Satura- } \\
\operatorname{tion}^{a}\end{array}$ & \multicolumn{2}{|c|}{$\begin{array}{l}\text { Pd ppt. in mg., } \\
\text { found }\end{array}$} & \multicolumn{2}{|c|}{${ }_{\text {Initial }}^{\text {Palladium Final }}$} & \multicolumn{2}{|c|}{$\underset{\text { Initial }}{\text { Potassium Final }}$} & $\begin{array}{l}\text { Soly. prod } \\
\times 108\end{array}$ \\
\hline & 1 & u & 125.0 & 125.2 & 18. 28 & 14.86 & 26.92 & 20.08 & 5.99 \\
\hline & 5 & $\mathrm{~s}$ & 125.3 & & & & & & \\
\hline & 8 & $\mathrm{~s}$ & 125.4 & & & & & & \\
\hline 2 & 8 & s & 95.2 & 95.2 & 13.28 & 11.30 & 26.92 & 22.96 & 5.96 \\
\hline & 11 & $\mathrm{~s}$ & 95.3 & & & & & & \\
\hline 3 & 1 & $\mathrm{u}$ & 50.6 & 50.7 & 8.80 & 6.02 & 37.04 & 31.48 & 5.97 \\
\hline & 3 & $\mathbf{u}$ & 50.7 & & & & & & \\
\hline & 5 & $\mathrm{~s}$ & 50.7 & & & & & & \\
\hline & 8 & $\mathrm{~s}$ & 50.9 & & & & & & \\
\hline 4 & 8 & s & 42.1 & 42.2 & 6.39 & 5.01 & 37.04 & 34.28 & 5.89 \\
\hline & 11 & $\mathrm{~s}$ & 42.4 & & & & & & \\
\hline 5 & 1 & $\mathbf{u}$ & 37.6 & 37.4 & 9.14 & 4.44 & 46.15 & 36.75 & 6.00 \\
\hline & 3 & $\mathbf{u}$ & 37.5 & & & & & & \\
\hline & 5 & $\mathrm{~s}$ & 37.2 & & & & & & \\
\hline & 8 & $\mathrm{~s}$ & 37.2 & & & & & & \\
\hline 6 & 8 & $\mathrm{~s}$ & 27.0 & 27.0 & 4.00 & 3.21 & 44.44 & 42.86 & 5.90 \\
\hline & 11 & $\mathrm{~s}$ & 27.0 & & & & & & \\
\hline 7 & 1 & $\mathbf{u}$ & 16.7 & 16.8 & 3.32 & 1.99 & 57.69 & 55.03 & 6.04 \\
\hline & 3 & $\mathbf{u}$ & 17.0 & & & & & & \\
\hline & 5 & $\mathrm{~s}$ & 16.7 & & & & & & \\
\hline & 8 & $\mathrm{~s}$ & 17.0 & & & & & & \\
\hline 8 & 8 & $\mathrm{~s}$ & 13.2 & 13.2 & 3.32 & 1.57 & 65.39 & 61.89 & 6.00 \\
\hline & 11 & $\mathrm{~s}$ & 13.2 & & & & & & \\
\hline
\end{tabular}

Mean value 5.97

${ }^{a}$ The letter $\mathrm{u}$ denotes previous undersaturation with $\mathrm{K}_{2} \mathrm{PdCl}_{6}$, which was attained by freezing it out at the start of the experiment. The letter s denotes previous supersaturation with $\mathrm{K}_{2} \mathrm{PdCl}_{6}$, attained by keeping the solution at $25^{\circ}$ from the start or by warming it to $40^{\circ}$ at the beginning.

The initial concentrations of palladium and potassium were known from the proportions of the stock solutions used in preparing the mixtures. The final palladium concentration was calculated from the weight given in the column headed "Pd ppt. in mg.," which shows the number of milligrams of the glyoxime compound obtained from $25.0 \mathrm{cc}$. of the saturated solution. Since some chloropalladate always crystallized out before equilibrium was attained, the final potassium concentration was found by di- 
rectly determining the decrease in the palladium content and correcting the initial potassium content accordingly. All these concentrations are given in the table in millimoles per liter. The values of the solubility product, $\left(\mathrm{K}^{+}\right)^{2}\left(\mathrm{PdCl}_{6}{ }^{--}\right)$, were calculated from the final molal concentrations of potassium and palladium. In this calculation all of the palladium was considered to be in the quadrivalent state in the form of the complex ion, $\mathrm{PdCl}_{6}{ }^{--}$, for the determinations of the equilibrium constant described below show that less than $0.5 \%$ is in the palladous state in a solution saturated with chlorine at atmospheric pressure.

It will be seen that, though the potassium concentration was altered three-fold and the palladium concentration thereby varied nine-fold, yet the solubility product remained nearly constant. The mean value of the solubility product at $25^{\circ}$ is $5.97 \times 10^{-6}$. This value, it should be noted, is that prevailing when the solvent is $1 N$ hydrochloric acid saturated with chlorine at $740-\mathrm{mm}$. pressure. ${ }^{6}$

\section{Equilibrium of the Oxidation Reaction in the Presence of Solid Potassium Chloropalladate}

The results of the experiments are presented in Table II. All of the concentrations are given in millimoles per liter, but in evaluating the equilibrium constant the concentrations were expressed in moles per liter.

In the first column is given the experiment number, to which is attached a letter $u$ or s showing the direction in which the equilibrium was approached. The letter $\mathrm{u}$ indicates that the solution was originally undersaturated with respect to potassium chloropalladate, for this salt was crystallized out at the start by passing undiluted chlorine through the solution in an ice-bath. The letter s denotes that the solution was originally supersaturated with chloropalladate, for the diluted chlorine was passed through the mixture in the thermostat from the start.

The second column shows the number of days during which the dilute chlorine was passed through the solution in the thermostat before taking the samples for analysis.

In the third column are given the concentrations of potassium in the equilibrium solution. These were derived from the initial values and from the decreases in palladium content determined as in the solubility experiments.

In the fourth column, headed "free chlorine," is given the concentration of free (uncombined) chlorine dissolved in the equilibrium solution. The value given is the concentration of chlorine in the acid solution increased by $2.2 \%$, to allow for the difference in the pressure of gas in the

6 The value corresponds to a solubility of 0.0114 molal, which is somewhat less than the solubility $\left(0.0179 \mathrm{~m}\right.$.) of potassium chloroplatinate in water at $25^{\circ}$ [Archibald, Wilcox and Buckley, This Journal, 30, 752 (1908)]. 
two pipets. The chlorine dissolved in the acid solution was determined by titrating with dilute thiosulfate the iodine liberated from potassium iodide.

In the fifth column, headed "total chlorine," is given the total oxidizing chlorine of the equilibrium solution. This was the value determined by the electrometric titration, for both the free chlorine and the chlorine combined with the quadrivalent palladium (as well as with any trivalent palladium) are reduced by the hydrazine.

In the sixth column, headed " $\mathrm{PdCl}_{6}{ }^{--}$obs.," is recorded one-half of the value obtained by subtracting from the total chlorine the free chlorine found in the pure acid solution. These values represent the concentration of this ion under the assumption that there is no trivalent palladium in the solution.

In the seventh column, headed " $\mathrm{PdCl}_{6}{ }^{--}$calcd.," are shown the concentrations of the chloropalladate ion as calculated from the mean value of the solubility product given in Table I. These should be the actual $\mathrm{PdCl}_{6}--$ concentrations provided the solubility equilibrium is attained.

In the eighth column are given the differences between the concentrations in the sixth and seventh columns. Except for experimental error these differences should be half the molal concentration of the $\mathrm{PdCl}_{5}^{--}$ if it also exists in the equilibrium mixture.

In the ninth column, headed "Total Pd," are given the concentrations of palladium obtained by the precipitation of the palladium with dimethylglyoxime after the solutions had been titrated with hydrazine for total chlorine.

In the tenth column under the heading " $\mathrm{PdCl}_{4}--$," are shown the concentrations of bivalent palladium as found from the difference between the palladium content given in the preceding column and the "observed

TABLE II

Equilibrium Data for the Oxidation Reaction in Normal Hydrochloric Acid in the Presence of Solid Potassium Chloropalinadate at $25^{\circ}$

\begin{tabular}{|c|c|c|c|c|c|c|c|c|c|c|}
\hline Expt. & $\begin{array}{l}\text { Time, } \\
\text { days }\end{array}$ & $\begin{array}{l}\text { Potas- } \\
\text { sium }\end{array}$ & $\begin{array}{c}\text { Free } \\
\text { chlorine }\end{array}$ & $\begin{array}{c}\text { Total } \\
\text { chlorine }\end{array}$ & $\begin{array}{l}\text { Millin } \\
\text { obs. }\end{array}$ & $\begin{array}{l}\text { oles of } \mathrm{P} \\
\text { calcd. }\end{array}$ & $\begin{array}{l}\mathrm{dCl}_{6}^{--} \\
\text {diff. }\end{array}$ & $\begin{array}{l}\text { Total } \\
\text { Pd }\end{array}$ & $\mathrm{PdCl}_{4}--$ & $\begin{array}{l}\text { Equil. } \\
\text { const. }\end{array}$ \\
\hline $1 \mathrm{u}$ & 1 & 49.4 & 0.117 & 2.66 & 2.54 & 2.45 & 0.09 & 8.60 & 6.06 & 3580 \\
\hline $2 u$ & 1 & 47.1 & .167 & 2.90 & 2.74 & 2.69 & .05 & 7.46 & 4.72 & 3480 \\
\hline $3 \mathrm{~s}$ & 3 & 46.9 & .184 & 3.10 & 2.92 & 2.71 & .21 & 7.35 & 4.43 & 3580 \\
\hline $4 \mathrm{~s}$ & 5 & 46.4 & .204 & 3.15 & 2.95 & 2.77 & .18 & 7.11 & 4.16 & 3480 \\
\hline $5 \mathrm{~s}$ & 1 & 45.6 & .282 & 3.61 & 3.33 & 2.87 & .46 & 6.70 & 3.37 & 3500 \\
\hline $6 u$ & 1 & 42.9 & .442 & 3.75 & 3.31 & 3.24 & .07 & 5.36 & 2.05 & 3650 \\
\hline $7 \mathrm{u}$ & 1 & 32.1 & 1.18 & 7.22 & 6.04 & 5.79 & .25 & 7.47 & 1.43 & 3580 \\
\hline $8 \mathrm{u}$ & 1 & 31.4 & 2.00 & 8.26 & 6.26 & 6.06 & .20 & 7.10 & 0.84 & 3740 \\
\hline $9 u$ & 5 & 28.3 & 2.04 & 9.13 & 7.59 & 7.43 & .16 & 8.57 & .98 & 3810 \\
\hline $10 u$ & 4 & 28.9 & 2.17 & 9.69 & 7.52 & 7.15 & .37 & 8.41 & .89 & 3900 \\
\hline $11 \mathrm{~s}$ & 1 & 28.7 & 2.63 & 10.13 & 7.50 & 7.25 & .25 & 8.26 & .76 & 3750 \\
\hline
\end{tabular}


$\mathrm{PdCl}_{6}{ }^{--}$" in the sixth column. The "calculated $\mathrm{PdCl}_{6}^{--}$" could not be used, since in many cases equilibrium with the solid was not attained.

In the last column are given the values for the equilibrium constant calculated by the expression $\left(\mathrm{PdCl}_{6}{ }^{--}\right) /\left(\mathrm{PdCl}_{4}{ }^{--}\right)\left(\mathrm{Cl}_{2}\right)$, using for the $\left(\mathrm{Cl}_{2}\right)$ the concentration of "free chlorine" and for $\left(\mathrm{PdCl}_{6}{ }^{-}\right)$the "observed" concentration given in the sixth column.

It will be seen from Table II that in every experiment the $\mathrm{PdCl}_{6}-$ concentration determined by the hydrazine titration is greater than that calculated from the solubility of potassium chloropalladate. This difference is smaller in Expts. 1, 2 and 6, but this may arise from the fact that only one day was allowed for the approach to equilibrium, and that therefore the solutions may have been still undersaturated with chloropalladate. The equilibrium between solution and solid was probably attained in the other experiments, where several days were allowed for the approach to equilibrium. There seems, therefore, always to be between the calculated and observed $\mathrm{PdCl}_{6}--$ concentrations a real difference, usually amounting to about 20 millimoles per liter, which seems greater than can be accounted for by experimental errors. This difference may be due to the presence in the solution of some trivalent palladium. ${ }^{7}$ At any rate the experiments prove that the amount of palladium present in the trivalent state is small compared with the amounts in the other two states in these solutions, and the concentration of it was neglected in the calculations of the equilibrium constant of the oxidation reaction. ${ }^{8}$

It will also be seen from Table II that the values of the equilibrium constant show an average deviation from the mean of only $3.2 \%$ and that the extreme deviation from the mean is $7.1 \% .^{9}$

\section{Equilibrium of the Oxidation Reaction with No Solid Phase Present}

The results of the equilibrium experiments where no solid phase was present are given in Table III. All the concentrations are given in millimoles per liter, but the equilibrium constant is again expressed in moles.

The first column gives the experiment number, to which an asterisk

7 The fact that the difference is substantially constant throughout the whole series of experiments is also in accord with the assumption that this difference is due to trivalent palladium; for, by the mass action law, $\left(\mathrm{PdCl}_{5}{ }^{--}\right) /\left(\mathrm{PdCl}_{6}{ }^{--}\right)=K /\left(\mathrm{Cl}_{2}\right)^{1 / 2}$, and it happens that in the experiments $\left(\mathrm{PdCl}_{6}{ }^{--}\right)$was approximately proportional to $\left(\mathrm{Cl}_{2}\right)^{1 / 2}$.

${ }^{8} \mathrm{It}$ might seem better, in view of the possible presence of trivalent palladium, to use for $\left(\mathrm{PdCl}_{6}{ }^{--}\right)$the values calculated from the solubility of the $\mathrm{K}_{2} \mathrm{PdCl}_{6}$; but the solubility equilibrium, unlike the equilibrium of the oxidation reaction, is established so slowly that saturation with the solid phase was not always reached.

9 The agreement between the individual results is of the order one would expect. For an error of $0.5 \mathrm{mg}$. in the gravimetric analysis for total palladium, in Expts. 1 and 11 for example, would make errors in the constant of 0.5 and $3.0 \%$, respectively, and an error of $0.2 \%$ in the electrometric titration would produce errors in the constant of 0.3 and $2.0 \%$, respectively. 
is attached when the experiment was made with the first palladium preparation, not with the final carefully purified one.

The second column shows the mean concentration of free chlorine as found from the titrations of the two pure acid solutions.

In the third column is given the concentration of total oxidizing chlorine found by the titration of the equilibrium mixture with hydrazine.

The fourth column, headed " $\mathrm{PdCl}_{6}--$," shows the concentration of quadrivalent palladium, under the assumption that this is equal to onehalf of the difference between the total and free chlorine.

TABLE III

Equilibrium Data for the Oxidation Reaction at $25^{\circ}$ with No Solid Phase A. Experiments with solutions $1 N$ in hydrochloric acid

\begin{tabular}{|c|c|c|c|c|c|c|c|}
\hline Expt. & $\begin{array}{c}\text { Free } \\
\text { chlorine }\end{array}$ & $\begin{array}{c}\text { Total } \\
\text { chlorine }\end{array}$ & $\mathrm{PdCl}_{6}^{--}$ & $\begin{array}{l}\text { Total } \\
\text { Pd }\end{array}$ & $\mathrm{PdCl}_{4}--$ & $\begin{array}{l}\text { Equil. } \\
\text { const. }\end{array}$ & $\begin{array}{l}\text { Per cent. } \\
\text { deviation }\end{array}$ \\
\hline 1 & 0.109 & 1.58 & 1.47 & 5.11 & 3.64 & 3700 & -0.3 \\
\hline $2^{*}$ & .131 & 3.29 & 3.16 & 9.40 & 6.24 & 3780 & +1.8 \\
\hline $3^{*}$ & .136 & 3.33 & 3.19 & 9.40 & 6.21 & 3780 & +1.8 \\
\hline 4 & .160 & 2.01 & 1.85 & 5.11 & 3.26 & 3550 & -4.3 \\
\hline 5 & .160 & 2.04 & 1.88 & 5.11 & 3.23 & 3640 & -1.8 \\
\hline 6 & .159 & 3.86 & 3.70 & 10.22 & 6.52 & 3570 & -3.8 \\
\hline 7 & .225 & 2.58 & 2.36 & 5.11 & 2.75 & 3810 & +2.7 \\
\hline 8 & .230 & 2.55 & 2.32 & 5.11 & 2.79 & 3620 & -2.4 \\
\hline $9 *$ & .496 & 6.68 & 6.18 & 9.40 & 3.22 & 3870 & +4.3 \\
\hline 10 & .537 & 7.27 & 6.73 & 10.22 & 3.49 & 3670 & -1.1 \\
\hline 11 & .538 & 7.34 & 6.80 & 10.22 & 3.42 & 3700 & -0.3 \\
\hline 12 & .564 & 4.02 & 3.46 & 5.11 & 1.65 & 3720 & +0.3 \\
\hline $13^{*}$ & .586 & 7.07 & 6.48 & 9.40 & 2.92 & 3790 & +2.2 \\
\hline 14 & .592 & 4.08 & 3.49 & 5.11 & 1.62 & 3720 & +0.3 \\
\hline 15 & .994 & 5.03 & 4.04 & 5.11 & 1.07 & 3800 & +2.4 \\
\hline 16 & 1.00 & 5.02 & 4.02 & 5.11; & 1.09 & 3690 & -0.5 \\
\hline 17 & 1.22 & 5.40 & 4.18 & 5.11 & 0.93 & 3680 & -0.8 \\
\hline 18 & 1.23 & 5.43 & 4.20 & 5.11 & 0.91 & 3750 & +1.1 \\
\hline
\end{tabular}

B. Experiments with solutions $0.3 \mathrm{~N}$ in hydrochloric acid and $0.7 \mathrm{~N}$ in sodium chloride

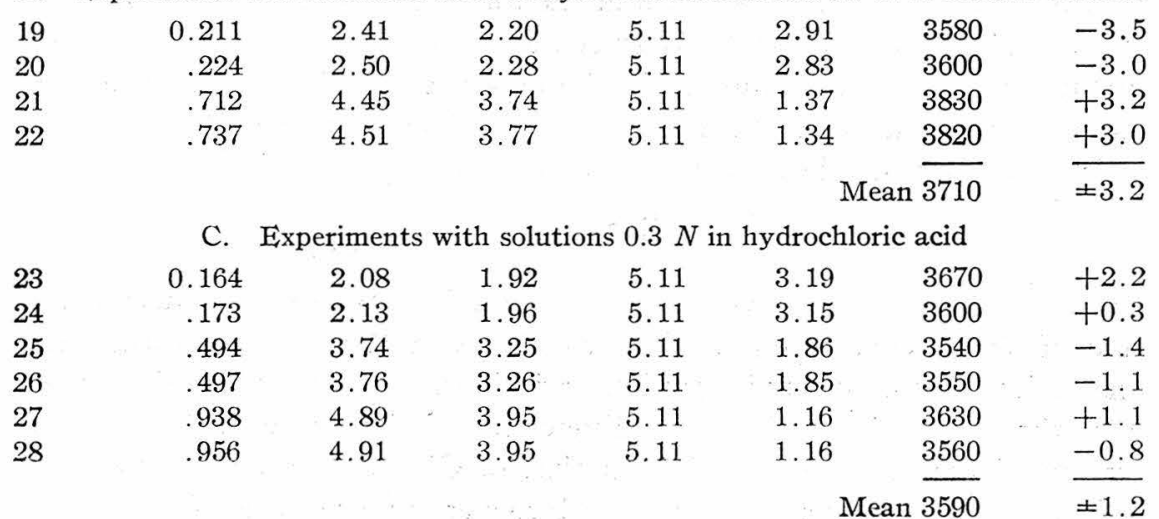


In the fifth column, under the heading "Total Pd," is given the total palladium content, calculated from the volume of the standard stock solution used in preparing the equilibrium mixture.

In the sixth column, under the heading " $\mathrm{PdCl}_{4}--$," is given the concentration of bivalent palladium found by subtracting the " $\mathrm{PdCl}_{6}{ }^{--}$" from the "Total Pd."

In the seventh column are given the equilibrium constants for the reaction as calculated from the molal concentrations of free chlorine, $\mathrm{PdCl}_{6}{ }^{--}$, and $\mathrm{PdCl}_{4}{ }^{--}$. In the last column are shown the percentage deviations of these values from the mean value.

\section{Discussion of the Equilibrium Data}

It will be seen from the experiments in Series A of Table III that, though the concentration of free chlorine was varied more than ten-fold and the concentration of palladium was doubled, yet the equilibrium constants show a maximum deviation from the mean of only $4.3 \%$, and an average deviation from the mean of only $1.8 \%$. Moreover the mean value, 3710 , of the constant differs only by $1.9 \%$ from the mean value, 3640 , obtained in the experiments recorded in Table II. We shall adopt 3710 for the equilibrium constant in normal hydrochloric acid solution, since the average deviation $(1.8 \%$ ) was considerably less than that $(3.2 \%)$ in the experiments of Table II.

The fact that the experimentally determined equilibrium constant does not vary with the concentration of free chlorine shows that little, if any, trivalent palladium is present in the solution; for its concentration would, in accordance with the mass action expression $\left(\mathrm{PdCl}_{5}^{--}\right) /\left(\mathrm{PdCl}_{4}^{--}\right)$ $=K^{\prime}\left(\mathrm{Cl}_{2}\right)^{1 / 2}$, be proportional to the square root of the chlorine concentration.

In the preceding considerations it has been assumed that the "free chlorine" is all present as chlorine molecules. It is, however, known that dissolved chlorine exists also in the form of hypochlorous acid and of trichloride ion. So long as the hydrogen-ion and chloride-ion concentrations in the solution are constant, as in the experiments of any one series, the various proportions of these three forms of chlorine will be constant, as is seen from the following expressions for the equilibria involved

$$
K_{\mathrm{H}}=\frac{\left(\mathrm{H}^{+}\right)\left(\mathrm{Cl}^{-}\right)(\mathrm{HClO})}{\left(\mathrm{Cl}_{2}\right)} \quad K_{\mathrm{C}}=\frac{\left(\mathrm{Cl}_{3}^{-}\right)}{\left(\mathrm{Cl}_{2}\right)\left(\mathrm{Cl}^{-}\right)}
$$

Change of the chlorine concentration should therefore not cause variation of the calculated equilibrium constants of the reaction within any one series, where the hydrogen-ion and chloride-ion concentrations were kept the same. Nevertheless, a divergence of the value of the concentration of the "free chlorine" from that of $\mathrm{Cl}_{2}$ as such would affect the numerical value of the constant. Moreover, in the case of the Series 
$\mathrm{A}, \mathrm{B}$ and $\mathrm{C}$, where the concentrations of hydrogen ion and chloride ion differ, such a divergence might cause a difference between the equilibrium constants of these three series. It is therefore necessary to consider the magnitudes of these effects of hydrolysis and complex ion formation.

From the value $4.84 \times 10^{-4}$ for $K_{\mathrm{H}}$ given by Lewis and Randall ${ }^{10}$ and from the activity coefficients of the hydrogen ion and chloride ion given by them (Ref. 10, p. 336) the ratio $(\mathrm{HClO}) /\left(\mathrm{Cl}_{2}\right)$ can be calculated. The calculation shows that in $1 \mathrm{~N}$ hydrochloric acid the hydrolysis of chlorine is entirely negligible (less than $0.1 \%$ ), and that even in $0.3 \mathrm{~N}$ hydrochloric acid it amounts to only $0.9 \%$, while in a solution $0.7 \mathrm{~N}$ in sodium chloride and $0.3 \mathrm{~N}$ in hydrochloric acid it is about $0.3 \%$.

The ratio of the concentration of $\mathrm{Cl}_{3}-$ to total $\mathrm{Cl}_{2}$ in these solutions may be found from the results obtained by Sherrill and Izard ${ }^{11}$ on the solubility of chlorine in solutions of hydrochloric acid and of sodium chloride. This ratio was found by them to be 0.154 in $1 N$ hydrochloric acid, and by interpolation it is found to be 0.049 in $0.3 \mathrm{~N}$ hydrochloric acid, and 0.150 in a solution $0.3 \mathrm{~N}$ in hydrochloric acid and $0.7 \mathrm{~N}$ in sodium chloride.

Moreover, the assumptions were made in calculating the equilibrium constants given above that the ratio of the concentrations of $\mathrm{PdCl}_{6}{ }^{--}$ and $\mathrm{PdCl}_{4}{ }^{--}$was equal to the ratio of their activities, and that the concentration of $\mathrm{Cl}_{2}$ was equal to its activity. The first of these assumptions is probably essentially correct, in view of the similarity of the two ions, but the activity coefficient of the chlorine is known to vary with change in the concentrations of hydrogen ion and chloride ion. Its activity coefficient is given by the ratio of the solubility of chlorine as such $\left(\mathrm{Cl}_{2}\right)$ in pure water to the solubility of chlorine in the equilibrium mixture. This is shown by the calculations of Sherrill and Izard to be 1.053 in $1 \mathrm{~N}$ hydrochloric acid and by interpolation is found to be 1.015 in $0.3 \mathrm{~N}$ hydrochloric acid and 1.238 in $0.7 \mathrm{~N}$ sodium chloride. Its activity coefficient in a solution $0.7 \mathrm{~N}$ in sodium chloride and $0.3 \mathrm{~N}$ in hydrochloric acid is 1.258 under the assumption that the salting-out effects of the hydrochloric acid and sodium chloride are additive.

In accordance with these considerations the concentration of chlorine as such $\left(\mathrm{Cl}_{2}\right)$ was determined by subtracting from the concentration of the "free chlorine" the concentrations of $\mathrm{HClO}$ and of $\mathrm{Cl}_{3}{ }^{-}$calculated from the ratios above; then its activity was found by multiplying the resulting concentration of $\mathrm{Cl}_{2}$ by the activity coefficient for chlorine in the equilibrium mixture. By using this activity of chlorine as such in place of the concentration of free chlorine, the values calculated from the three

${ }^{10}$ Lewis and Randall, "Thermodynamics," p. 508, calculated this value from the work of Jakowkin.

11 Sherrill and Izard, private communication concerning a research at the Massachusetts Institute of Technology, 1929. 
mean values given in Table III for the equilibrium constant of the oxidation reaction are found to be 4160 in $1 N$ hydrochloric acid, 3480 in a solution $0.7 \mathrm{~N}$ in sodium chloride and $0.3 \mathrm{~N}$ in hydrochloric acid, and 3750 in $0.3 \mathrm{~N}$ hydrochloric acid.

It will be seen that a 3.3 -fold decrease of the concentration of the hydrogen ion, while that of the chloride ion is kept constant, does not have a large effect on the equilibrium constant. This indicates that little of the palladium in these solutions is in the form of hydrolyzed acids, such as $\mathrm{H}_{2} \mathrm{PdCl}_{2}(\mathrm{OH})_{2}$ or $\mathrm{H}_{2} \mathrm{PdCl}_{5}(\mathrm{OH})$, or in the state of un-ionized acids.

It will also be seen that a 3.3-fold decrease in the concentration of the chloride ion, while that of the hydrogen ion is kept constant, causes only a small change in the mean value of the equilibrium constant. This fact proves that the bivalent palladium and the quadrivalent palladium must both be nearly or quite completely in the form of such complex ions as result from the addition of the same number of chloride ions to the simple chlorides $\left(\mathrm{PdCl}_{2}\right.$ and $\left.\mathrm{PdCl}_{4}\right)$; for otherwise the chloride-ion concentration would appear in the equilibrium expression and would greatly affect the value of the constant calculated as above without taking this into account. In view of the solid salts known to be formed from quadrivalent palladium in chloride solution and of its analogy with quadrivalent platinum, there is therefore little doubt that the two complex ions present are almost wholly $\mathrm{PdCl}_{6}{ }^{--}$and $\mathrm{PdCl}_{4}^{--}$

The Reduction Potential of Bivalent-Quadrivalent Palladium in Chloride Solutions

The free-energy decrease $-\Delta F$ attending the reaction $\mathrm{PdCl}_{4}{ }^{--}+$ $\mathrm{Cl}_{2}=\mathrm{PdCl}_{6}{ }^{--}$when all the substances have an activity of 1.0 molal, is found by the formula $-\Delta F=R T \ln K$ to be 20,620 joules, using for the equilibrium constant $K$ the corrected value 4160 in $1.0 \mathrm{~N}$ hydrochloric acid. The corresponding potential $\mathrm{E}$ obtained from the expression. $\mathrm{E}=$ $-\Delta F / 2 \times 96,500$ is 0.1070 volt. For the case that the chlorine has a partial pressure of one atmosphere the potential for the reaction is 0.0707 volt. This is found by correcting the potential by $1 / 2(R T / F) \ln c$ (or $0: 0296 \log c)$, where $c$ represents the solubility (0.0592 molal) in water of chlorine gas when its partial pressure is one atmosphere.

The equation for the free energy is therefore

$$
\mathrm{PdCl}_{4}{ }^{--}(1 \mathrm{~m} .)+\mathrm{Cl}_{2}(1 \mathrm{~atm} .)=\mathrm{PdCl}_{6}--(1 \mathrm{~m} .)+2 \mathrm{~F} \times 0.0707 \text { joule }
$$

The following equation expresses the reduction potential of $\mathrm{Cl}_{2}$ (1 atm.) $\mathrm{Cl}^{-}\left(1 \mathrm{~m}\right.$.), when $\mathrm{E}^{-}$represents one mole of electrons

$$
2 \mathrm{Cl}^{-}(1 \mathrm{~m} .)=\mathrm{Cl}_{2}(1 \mathrm{~atm} .)+2 \mathrm{E}^{-}-2 \mathrm{~F} \times 1.359 \text { joules }
$$

Adding these two equations, one obtains, when all three ions are 1.0 molal

$$
\mathrm{PdCl}_{4}{ }^{--}+2 \mathrm{Cl}^{-}=\mathrm{PdCl}_{6}^{--}+2 \mathrm{E}^{-}-2 \mathrm{~F} \times 1.288 \text { joules }
$$


The reduction potential of $\mathrm{PdCl}_{4}--+2 \mathrm{Cl}^{-}$(reduced state) with respect to $\mathrm{PdCl}_{6}--$ (oxidized state) is therefore -1.288 volts at $25^{\circ}$.

\section{Summary}

Measurements have been made of the solubility at $25^{\circ}$ of potassium chloropalladate $\left(\mathrm{K}_{2} \mathrm{PdCl}_{6}\right)$ in normal hydrochloric acid containing potassium chloride and saturated with chlorine at atmospheric pressure, and the value $5.97 \times 10^{-6}$ has thus been found for the solubility product $\left(\mathrm{K}^{+}\right)^{2}\left(\mathrm{PdCl}_{6}{ }^{--}\right)$in moles per liter.

The equilibrium of the oxidation reaction $\mathrm{PdCl}_{4}{ }^{--}+\mathrm{Cl}_{2}=\mathrm{PdCl}_{6}{ }^{--}$ has been studied. This was first done by using normal hydrochloric acid solutions of palladium chloride saturated both with solid potassium chloropalladate and with chlorine hundred-fold diluted with nitrogen. A comparison of the $\mathrm{PdCl}_{6}--$ concentration calculated from the solubility product for $\mathrm{K}_{2} \mathrm{PdCl}_{6}$ with that derived from the analytically determined concentration of combined oxidizing chlorine showed that little, if any, trivalent palladium exists in the equilibrium mixture.

More exact measurements were also made of the equilibrium of the oxidation reaction without any solid phase present. The value 4160 was thus found for the equilibrium constant $K=\left(\mathrm{PdCl}_{6}{ }^{--}\right) /\left(\mathrm{PdCl}_{4}^{--}\right) /\left(\mathrm{Cl}_{2}\right)$ in $1 \mathrm{~N}$ hydrochloric acid solution, using in the expression the molal concentrations of bivalent and quadrivalent palladium and the activity of chlorine as such, obtained by subtracting from the total free chlorine the calculated concentrations of $\mathrm{HClO}$ and $\mathrm{Cl}_{3}-$ present and by changing the resulting concentrations of $\mathrm{Cl}_{2}$ over to activities with the aid of the relative solubilities of chlorine gas in water and in hydrochloric acid.

Values were also found for the equilibrium constant in $0.3 \mathrm{~N}$ hydrochloric acid solution, and in a solution $0.3 \mathrm{~N}$ in hydrochloric acid and 0.7 $N$ in sodium chloride. These values, corrected as just described, were 3750 and 3480 , respectively. This shows that these variations in the chloride-ion and hydrogen-ion concentrations do not have a large effect on the equilibrium, and therefore proves that the two complex ions present are those formed from the simple chlorides, $\mathrm{PdCl}_{2}$ and $\mathrm{PdCl}_{4}$, by the addition of the same number of chloride ions, and that they are therefore doubtless $\mathrm{PdCl}_{4}{ }^{--}$and $\mathrm{PdCl}_{6}{ }^{--}$; also that little of the palladium is in the form of hydrolyzed acids, such as $\mathrm{H}_{2} \mathrm{Pd}(\mathrm{OH})_{2} \dot{\mathrm{Cl}}_{2}$ or $\mathrm{H}_{2} \mathrm{Pd}(\mathrm{OH}) \mathrm{Cl}_{5}$.

From the equilibrium constant (4160) in normal hydrochloric acid, the reduction potential for the reaction $\mathrm{PdCl}_{4}{ }^{--}+2 \mathrm{Cl}^{-}=\mathrm{PdCl}_{6}^{--}+2 \mathrm{E}^{-}$ at $25^{\circ}$ is computed to be $-\mathbf{1 . 2 8 8}$ volts.

Pasadena, California 